\title{
Injection terahertz laser using the resonant inter-layer radiative transitions in double-graphene-layer structure
}

\author{
V. Ryzhii ${ }^{1,2}$, A. A. Dubinov ${ }^{3}$, V. Ya. Aleshkin ${ }^{3}$, M. Ryzhii ${ }^{2,4}$, and T. Otsuji ${ }^{1,2}$ \\ ${ }^{1}$ Research Institute for Electrical Communication, \\ Tohoku University, Sendai 980-8577, Japan \\ 2 Japan Science and Technology Agency, \\ CREST, Tokyo 107-0075, Japan \\ ${ }^{3}$ Institute for Physics of Microstructures of Russian Academy of Sciences, \\ and Lobachevsky State University of Nizhny Novgorod, \\ Nizhny Novgorod 603950, Russia \\ ${ }^{4}$ Department of Computer Science and Engineering, \\ University of Aizu, Aizu-Wakamatsu 965-8580, Japan
}

\begin{abstract}
We propose and substantiate the concept of terahertz $(\mathrm{THz})$ laser enabled by the resonant electron radiative transitions between graphene layers (GLs) in double-GL structures. We estimate the $\mathrm{THz}$ gain for TM-mode exhibiting very low Drude absorption in GLs and show that the gain can exceed the losses in metal-metal waveguides at the low end of the $\mathrm{THz}$ range. The spectrum of the emitted photons can be tuned by the applied voltage. A weak temperature dependence of the $\mathrm{THz}$ gain promotes an effective operation at room temperature.
\end{abstract}

The gapless energy spectrum of graphene layers (GLs) 1] enables the creation of different terahertz (THz) devices utilizing the interband transition. In particular, the interband population inversion and the pertinent negativity of the dynamic conductivity in GLs [2, 3] due to the optical or injection pumping can be used in GL-based $\mathrm{THz}$ lasers [4 9]. First experimental results on the $\mathrm{THz}$ emission from optically excited GLs [10] (see also review paper [11] and references therein) instill confidence in the realization of such lasers. One of the obstacles, limiting the achievement of the negative dynamic conductivity in the range of a few $\mathrm{THz}$, is the reabsorption of the photons with the in-plane polarization emitted at the interband transitions due to the intraband transitions (the Drude absorption). Similar situation takes place in the quantum cascade lasers (QCLs) based on multiple quantum well (MQW) structures [12]. However, in the case of the photon polarization perpendicular to the QW plane the intraband (intrasubband) absorption can be much weaker than that following from the semi-classical Drude formula [13].

In this paper, we propose a device structure based on a double-GL structure shown in Fig. 1 (upper panel) with the injection of electrons to one n-doped GL and to another p-doped GL, which can be used for lasing of $\mathrm{THz}$ photons with the electric field perpendicular to the GL plane due to the tunneling inter-GL radiative processes. The structure band diagram under the applied bias voltage and tunneling transitions assisted with the emission of photons with the energy $\hbar \omega \sim \Delta$, where $\Delta$ is the energy distance (gap) between the Dirac points in GLs, are demonstrated in Fig. 1 (lower panel). These transitions take place from the conduction band of the GL with 2DEG to the empty conduction band of GL with 2DHG. The transition from the filled valence band the former GL to the empty portion of the valence band of the latter GL also contribute to the emission of photons with $\hbar \omega \sim \Delta$. The structure comprises two GLs with the side contact at one of the GL edges. This double-GL structure plays the role of the laser active region. The opposite edge of GL is isolated from another contact. A narrow tunneling-transparent barrier separates GLs (its thickness $d$ is about few nanometers). The applied bias voltage $V$ provides the formation of the two-dimensional electron and hole gases (2DEG and 2DHG) in the upper and lower GLs, respectively owing to the injection from the side contacts, so that the inter-GL population inversion occurs. The GL structures in question were fabricated recently [14 17]. The effective control of the GL population was, in particular, used for modulation of optical radiation [14, 15]. These structures can exhibit marked inter-GL tunneling current, which in the case of the Dirac point alignment, exhibit the negative differential inter-GL conductivity [18] (see also Refs. 19 21], where the latter effect was theoretically considered). The inter-GL barrier layers can be made of $\mathrm{hBN}, \mathrm{WS}_{2}$, or similar materials. The double-GL structures can also be used in different devices ( $\mathrm{THz}$ detectors and photomixers) utilizing the plasmonic effects 22 24].

The $\mathrm{THz}$ laser metal-metal (MM) waveguide system consist of two parallel metal strips (see Fig. 1, upper panel) as in some QCLs [12]. The net spacing between the strips $(2 W+d)$ is about $10 \mu \mathrm{m}$. The spatial distributions of the amplitudes of $\mathrm{THz}$ electric-field components $\left|E_{z}\right|$ and $\left|E_{x}\right|$ in the TM-mode propagating in the $y$-direction and the real part of refractive index in the MM waveguide are shown in Fig. 2. It is assumed that the device structure is supplied with the proper mirrors reflecting the radiation propagating in the $y$-direction.

To enhance the laser output power, a more complex active region can used which includes several doubleGL structures (with thin inter-GL layers and relatively thick layers separating the double-GL structures) or a multiple-GL structure with the tunneling-transparent 

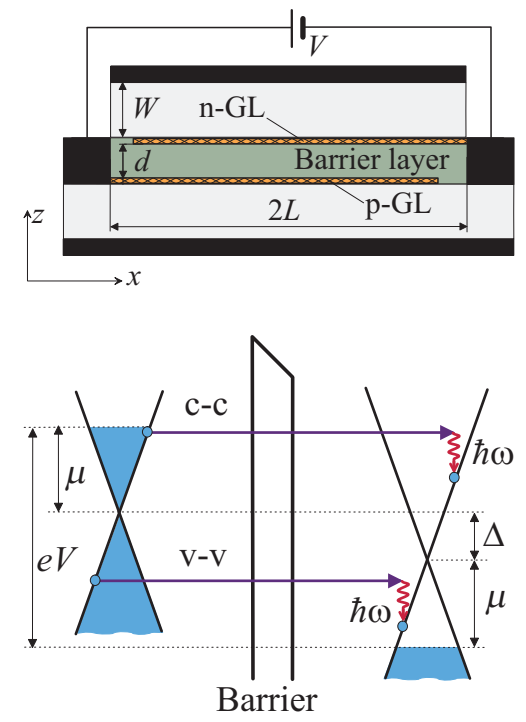

FIG. 1: Schematic view of the DG-laser with double-GL structure and MM waveguide(upper panel) and the device band diagram (lower panel). Dark regions correspond to the highly conducting side contacts and metal wave-guide strips (along the $y$-direction. Arrows in the lower panel indicate the resonant-tunneling (with the conservation of the electron momentum) inter-GL transitions between the conduction band $(c-c)$ and the valence band $(v-v)$ states and assisted by the emission of photons (at weak depolarization shift).

inter-GL barrier layers. A wave guide system using the specific plasmon-polaritons [25] (see also Ref.[7]), associated the high conductivity of GLs and propagating along the structure (in the $y$-direction) can also be implemented.

Apart from weaker intraband reabsorption of the generated photons, the double-GL structures (and more complex ones) might exhibit the advantages in comparison with the p-i-n lasers with simultaneous injection of both electrons and holes into the same GLs [ $8,26,27]$ because of a more effective injection due to the absence of the electron-hole "friction" [28] and the weakening of nonradiative recombination mechanisms, in particular, that associated with the emission of optical phonons.

The electron and hole density in the pertinent GLs is given by

$$
\Sigma=\Sigma_{i}+\frac{\kappa \Delta}{4 \pi e^{2} d}
$$

where $\Sigma_{i}$ is the density of donors in the upper GL (n-GL) and acceptors in the lower GL (p-GL), $\kappa$ is the dielectric constant, and $e=|e|$ is the electron charge. If the length of the electron and hole diffusion exceeds the length of GLs, the local potential difference between GLs is close to $V$ [8, 27]. In this situation, the quantity $\Delta$ (which determines the electric field $E=\Delta / e d$ in the inter-GL barrier) obeys the following equation (see Fig. 1):

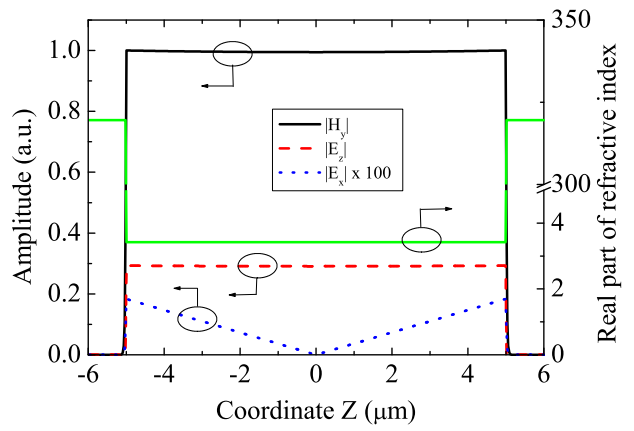

FIG. 2: Spatial distributions of the amplitudes of $\mathrm{THz}$ electric- and magnetic-field components in TM mode and the real part of the refraction index in the MM waveguide under consideration.

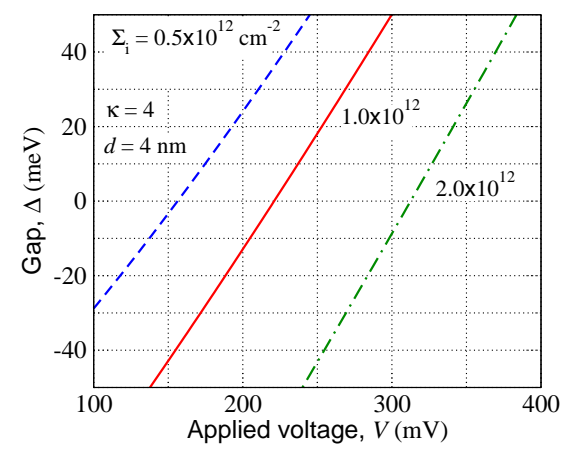

FIG. 3: Energy gap between Dirac points $\Delta$ versus applied voltage $V$ at different dopant densities $\Sigma_{i}$.

$$
\Delta+2 \mu=e V
$$

where $\mu$ is the value of the Fermi energy of 2DEG and $2 \mathrm{DHG}$ in the pertinent GL. In the case of the 2DEG and 2DHG strong degeneration $(\mu \gg T$, where $T$ is the temperature in the energy units),

$$
\mu \simeq \hbar v_{W} \sqrt{\pi \Sigma}
$$

respectively, where $v_{W} \simeq 10^{8} \mathrm{~cm} / \mathrm{s}$ is the characteristic velocity of electrons and holes in GLs. Considering Eqs. (1) - (3), in the above limiting cases, we arrive at

$$
\Delta / e=V+V_{0}-\sqrt{2 V V_{0}+V_{0}^{2}+V_{t}^{2}},
$$

where $V_{0}=\hbar^{2} v_{W}^{2} \kappa / 2 e^{3} d$ and $V_{t}=2 \hbar v_{W} \sqrt{\pi \Sigma_{i}} / e$ At $V \leq V_{t}, \Delta \leq 0$, while at $V>V_{t}, \Delta>0$. The band diagram at the bias voltage $V>V_{t}$ is shown in Fig. 1 (lower panel). In this case, the tunneling inter-GL transitions are possible only due to the scattering processes 
accompanying the tunneling [19 21]. However, the interGL transitions assisted by the emission of photons with the polarization corresponding to the photon electric field perpendicular to GLs (along the axis $z$ ) conserve the electron momentum and, hence, do not require any scattering (resonant-tunneling photon-assisted transitions). Assuming $\kappa=4, d=4 \mathrm{~nm}$, and $\Sigma_{i}=10^{12} \mathrm{~cm}^{-2}$, one obtains $V_{0} \simeq 136 \mathrm{mV}$ and $V_{t} \simeq 221 \mathrm{mV}$. The quantities $\Delta=5-10 \mathrm{meV}$ correspond to $V \simeq 229-237 \mathrm{mV}$ and $\mu \simeq 112.0-113.5 \mathrm{meV}$.

The real part of the transverse ac conductivity, $\operatorname{Re} \sigma_{z z}(\omega)$, of the double-GL structure under consideration can be estimated using the following formula:

$$
\operatorname{Re} \sigma_{z z}(\omega)=-\frac{2 e^{2}}{\hbar} \frac{\left|z_{u, l}\right|^{2} \Sigma_{i}\left(1+\Delta / \Delta_{i}\right) \gamma \hbar \omega}{\left[\hbar^{2}\left(\omega-\omega_{\max }\right)^{2}+\gamma^{2}\right]}
$$

Here

$$
\hbar \omega_{\max }=\Delta-\frac{8 \pi e^{2}\left|z_{u, l}\right|^{2} \Sigma_{i}\left(1+\Delta / \Delta_{i}\right)}{\kappa d}
$$

is the inter-GL resonant transition energy, where the second term in the right-hand side constitutes the depolarization shift(see, for instance, Ref. 29]), which for media with the population inversion is negative, $z_{u, l}=\int \varphi_{u}^{*}(z) z \varphi_{l}(z)$, where $\varphi_{u}(z)$ and $\varphi_{l}(z)$ are the $z$-dependent factors of the wave functions in the upper and lower GLs, $\gamma$ is the relaxation broadening, and $\Delta_{i}=4 \pi e^{2} d \Sigma_{i} / \kappa$.

Figure 3 shows the $\Delta$ versus $V$ dependences calculated using Eq. (4) at $\kappa=4, d=4 \mathrm{~nm}$, and different values of the dopant densities $\Sigma_{i}$.

At the resonance $\hbar \omega=\hbar \omega_{\max }$, Eq. (5) yields

$$
\operatorname{Re} \sigma_{z z}(\omega)=-\frac{2 e^{2}\left|z_{u, l}\right|^{2} \Sigma_{i}\left(1+\Delta / \Delta_{i}\right)}{\hbar}\left(\frac{\hbar \omega_{\max }}{\gamma}\right) .
$$

Introducing the gain-overlap factor for the $\mathrm{THz}$ mode in the wave guide under consideration $\Gamma=\left[\int_{-L}^{L} d x\left|E_{z}(x, 0)\right|^{2} / \int_{-L}^{L} \int_{-W}^{W} d x d z\left|E_{z}(x, 0)\right|^{2}\right]$, where $E_{z}(x, z)$ is the spatial distribution of the $\mathrm{THz}$ electric field in the TM mode propagating along the MM waveguide (in the $y$ direction) and $2 L$ is the length of GLs (distance between t he side contacts), the maximum THz gain $g=4 \pi \operatorname{Re} \sigma \Gamma / c \sqrt{\kappa}$, where $c$ is the speed of light in vacuum, can be estimated as

$$
g=\frac{8 \pi e^{2}\left|z_{u, l}\right|^{2} \Sigma_{i}\left(1+\Delta / \Delta_{i}\right)}{\hbar c \sqrt{\kappa}}\left(\frac{\hbar \omega_{\max }}{\gamma}\right) \Gamma
$$

The value of matrix element $\left|z_{u, l}\right|^{2}$ strongly depends on $d$ and $\Delta$. It is evaluated using a simple model, in which each GL is considered as a delta-layer separated by a barrier layer made of $\mathrm{WS}_{2}$. Following Ref. [19], we assume that the conduction band offset between a GL and $\mathrm{WS}_{2}$ barrier and the effective electron mass in

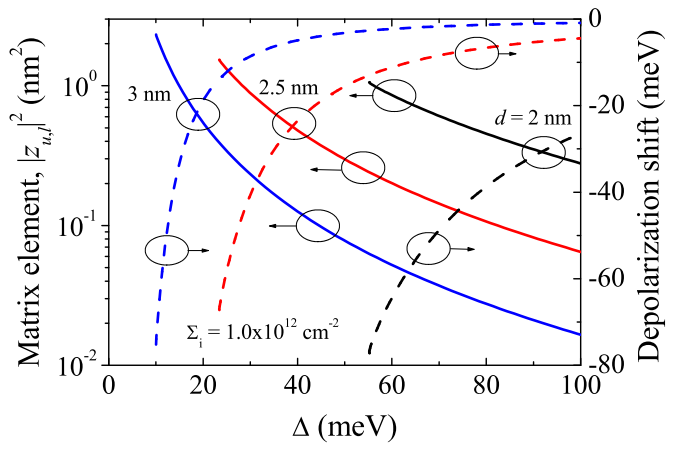

FIG. 4: Inter-GL matrix element $\left|z_{u, l}\right|^{2}$ (solid lines) and depolarization shift (dashed lines) as a function of gap between the Dirac points in GLs $\Delta$.

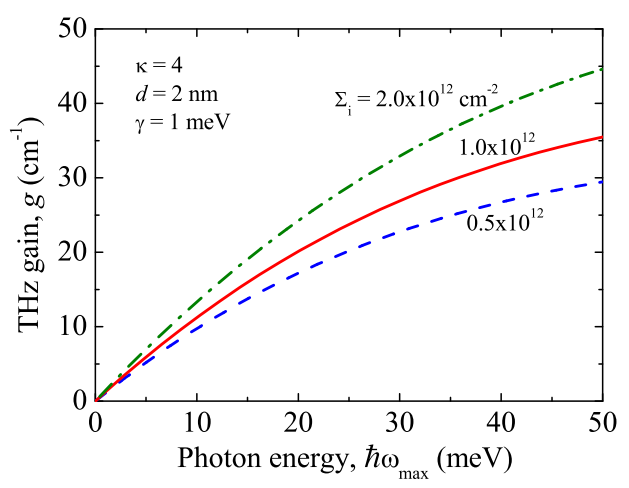

FIG. 5: THz gain $g$ versus the resonant photon energy $\hbar \omega_{\max }$ for double-GL structures with different dopant densities $\Sigma_{i}$ and $d=2 \mathrm{~nm}$.

$\mathrm{WS}_{2}$ are equal to $0.4 \mathrm{eV}$ and 0.27 of the free electron mass, respectively [29]. Figure 4 shows the dependence of the matrix element $\left|z_{u, l}\right|^{2}$ on the energy difference between the Dirac points $\Delta$ and the value of the depolarization shift (dependent of $\left|z_{u, l}\right|^{2}$ ) for different thicknesses of the $\mathrm{WS}_{2}$ inter-GL barrier. As seen from Fig. $4,\left|z_{u, l}\right|^{2}$ dramatically decreases with increasing $\Delta$. According to Eqs. (5) - (8), this substantially affects the transverse ac conductivity $\operatorname{Re} \sigma_{z z}(\omega)$, the resonant value of the photon energy $\hbar \omega_{\max }$, and, hence the $\mathrm{THz}$ gain $g$.

Figures 5 and 6 show the resonant $\mathrm{THz}$ gain versus photon energy $\hbar \omega_{\max }$ for different dopant densities $\Sigma_{i}$ and the inter-GL barrier layer thicknesses. In Fig. (5), it is assumed that $\kappa=4, d=2 \mathrm{~nm}$ and $\gamma / \hbar=1.6 \times 10^{12} \mathrm{~s}^{-1}$. The gain-overlap factor for the MM waveguide with $W=5 \mu \mathrm{m}$ is set to be $\Gamma=10^{3} \mathrm{~cm}^{-1}$. As seen from Fig. 5, an increase in the dopant density naturally increases the $\mathrm{THz}$ gain. However, this increase can be limited by an increase in broadening parameter $\gamma$. 


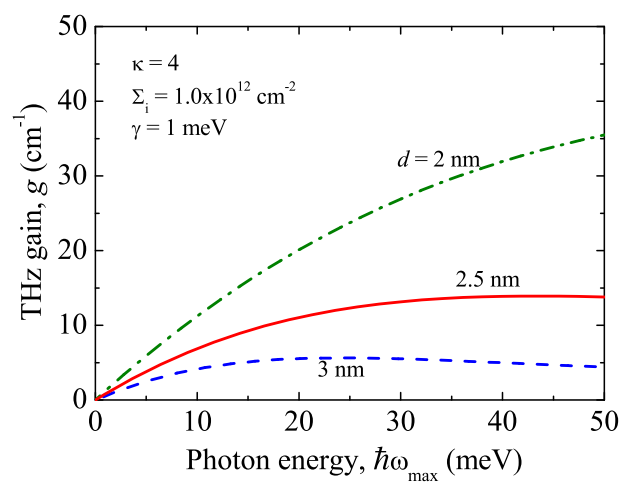

FIG. 6: The same as in Fig. 5 but for different $d$ and $\Sigma_{i}=$ $1.0 \times 10^{12} \mathrm{~cm}^{-2}$.

One can also see that the $\mathrm{THz}$ gain exceeds (or even well exceeds) the value $10 \mathrm{~cm}^{-1}$ in the range $\hbar \omega_{\max } \gtrsim 10 \mathrm{meV}$ $\left(\omega_{\max } / 2 \pi \gtrsim 2.5 \mathrm{THz}\right)$. Hence, $g$ can exceed the TMmode losses $\alpha$ in the copper MM waveguides even at room temperature 31]. It is worth noting that the Drude absorption for the mode under consideration can be very weak because of relatively small $\mathrm{THz}$ electric field component $E_{x}$ (as seen from Fig.2, the ratio $\left|E_{z}\right| /\left|E_{x}\right|>100$ ), particularly at $z=0$, where the double-GL structure is placed. The $\mathrm{THz}$ gain of the TM mode in the laser under consideration is at least comparable the maximum $\mathrm{THz}$ gain (without the Drude losses) in the injection lasers utilizing the TE mode and the intra-GL radiative transitions (see, for instance, Ref. [6]). Hence the former device can exhibit advantages due to the effective suppression of the Drude absorption. As follows from Eq. (8), the $\mathrm{THz}$ gain weakly depends on the temperature. Some temperature dependence appears due to the temperature dependence of parameter $\gamma$ ) or at low doping levels when $\mu \lesssim T)$. Weak temperature dependence of the resonant tunneling in double-GL structures was observed experi- mentally [18] (see also Refs. [19, 20]). This can provide the superiority of the double-GL lasers under consideration over the GL-based lasers using the intra-GL transitions discussed previously [4 6] and QCL lasers [12, 31] at elevated temperatures in the low end of the $\mathrm{THz}$ range as well as in the range where the operation of QCLs is hampered by the optical phonon absorption.

The $\mathrm{THz}$ gain in the double-GL structures with thicker inter-GL layer is markedly smaller as seen from Fig. 6. In this case, to overcome the waveguide losses the device structure with an active region consisting of a system of several parallel double-GL structures can be used. Each the double-GL system can be separated from others by relatively thick, non-transparent barrier layer (with the thickness $D \gg d$. In such a case, the quantity $g$ for the $\mathrm{THz}$ gain given by Eq. (8) should be multiplied by the number of the double-GL systems in the device structure. The multiple-GL structures in question can provide a much higher net $\mathrm{THz}$ gain without a marked increase in the Drude absorption enabling room temperature $\mathrm{THz}$ lasing in the range $\hbar \omega_{\max } \simeq 5-10 \mathrm{meV}$ $\left(\omega_{\max } / 2 \pi \simeq 1.25-2.5 \mathrm{THz}\right)$, where they might compete with the $\mathrm{THz}$ oscillators based on $\mathrm{A}_{3} \mathrm{~B}_{5}$ resonanttunneling structures [32].

In conclusions, we proposed $\mathrm{THz}$ lasers based on double-GL structures using the inter-GL radiative transitions and estimated their $\mathrm{THz}$ gain at different photon energies and applied voltages. It was demonstrated that the laser can exhibit advantages over other $\mathrm{THz}$ lasers due to the essential suppression of the Drude absorption in GL, weak temperature dependence of the $\mathrm{THz}$ gain even at room temperature, and voltage tuning of the spectrum of the emitted photons.

The authors are grateful to A. Satou and D. Svintsov for useful discussions. This work was supported by the Japan Society for Promotion of Science (Grantin-Aid for Specially Promoting Research, No.23000008) and the Japan Science and Technology Agency (CREST Project)), Japan, as well as by the Russian Foundation of Basic Research and the Dynasty Foundation, Russia.
[1] A. H. Castro Neto, F. Guinea, N. M. R. Peres, K. S. Novoselov, and A. K. Geim, Rev. Mod. Phys. 81, 109 (2009).

[2] V. Ryzhii, M. Ryzhii, and T. Otsuji, J. Appl. Phys. 101, 083114 (2007).

[3] F. Rana, IEEE Trans. Nanotechnol. 7, 91 (2008).

[4] A. A. Dubinov, V. Ya. Aleshkin. M. Ryzhii, T. Otsuji, and V. Ryzhii, Appl. Phys. Express 2, 092301 (2009).

[5] V. Ryzhii, M. Ryzhii, A. Satou, T. Otsuji, A. A. Dubinov, and V. Ya. Aleshkin, J. Appl. Phys. 106, 084507 (2009).

[6] V. Ryzhii, A. A. Dubinov, T. Otsuji, V. Mitin, and M. S. Shur, J. Appl. Phys. 107, 054505 (2010).

[7] A. A. Dubinov, V. Ya. Aleshkin, V. Mitin, T. Otsuji, and V. Ryzhii, J. Phys.: Condens. Matter 23, 141302 (2011).

[8] V. Ryzhii, M. Ryzhii, V. Mitin, and T. Otsuji, J.
Appl.Phys. 110, 094503 (2011).

[9] V. V. Popov, O. V. Polischuk, A. R. Davoyan, V. Ryzhii, T. Otsuji, and M. S. Shur, Phys. Rev. B 86, 195437 (2012).

[10] S. Boubanga-Tombet, S. Chan, T. Watanabe, A. Satou, V. Ryzhii, and T. Otsuji, Phys. Rev. B 85, 035443 (2012).

[11] T. Otsuji, S. A. Boubanga Tombet, A. Satou, M. Ryzhii, and V.Ryzhii, IEEE J. Select Topics in Quant. Electron. 19, 8400209 (2013).

[12] B. S. Williams, Nature photonics, 1, 517 (2007).

[13] F. Carosella, C. Ndebeka-Bandou, R. Ferreira, E. Dupont, K. Unterrainer, G. Strasser, A. WAcker, and G. Bastard, Phys. Rev. B 85, 085310 (2012).

[14] M. Liu, X. Yin, E. Ulin-Avila, B. Geng, T. Zentgraf, L. Ju, F. Wang, and X. Zhang: Nature, 474, 64 (2011). 
[15] M. Liu, X. Yin, and X. Zhang, Nano Lett. 12,1482 (2012).

[16] L. Britnell, R. V. Gorbachev, R. Jalil, B.D . Belle, F.Shedin, A. Mishenko, T. Georgiou, M. I. Katsnelson, L. Eaves, S. V. Morozov, N. M. R. Peres, J. Leist, A. K. Geim, K. S. Novoselov, and L. A. Ponomarenko, Science, 335,947 (2012).

[17] T. Georgiou, R. Jalil, B. D. Bellee, L. Britnell, R. V. Gorbachev, S. V. Morozov, Y.-J. Kim, A. Cholinia, S. J. Haigh, O. Makarovsky, L. Eaves, L. A. Ponimarenko, A. K. Geim, K. S. Nonoselov, and A. Mishchenko, Nature Nanotechnology 7, 100 (2013).

[18] L. Britnell, R. V. Gorbachev,A. K. Geim, L. A. Ponimarenko,A. Mishchenko, M. T. Greenaway, T. M. Fromhold, K. S. Nonoselov, and L. Eaves, Nature Comm. 4, 1794 (2013).

[19] R. M. Feenstra, D. Jena, and G. Gu: J. Appl. Phys 111, 043711 (2012).

[20] P. Zhao, R. M. Feenstra, G. Gu, D. Jena: IEEE Trans. Electron Devices 60, 951 (2012).

[21] F. T. Vasko: Phys. Rev.B 87, 075424 (2013).

[22] V. Ryzhii, T. Otsuji, M. Ryzhii, and M. S. Shur, J. Phys. D: Appl. Phys. 45, 302001 (2012).

[23] V. Ryzhii, A. Satou, T. Otsuji, M. Ryzhii, V. Mitin, and
M. S. Shur, J. Phys. D: Appl. Phys. 46 (2013) 315107.

[24] V. Ryzhii, M. Ryzhii, V. Mitin, M. S. Shur, A. Satou, and T. Otsuji: J. Appl. Phys. 113, 174506 (2013).

[25] D. Svintsov, V. Vyurkov, V. Ryzhii, and T. Otsuji, J. Appl. Phys. 113, 053701 (2013).

[26] M. Ryzhii and V. Ryzhii, Jpn. J. Appl. Phys. 46, L151 (2007).

[27] V. Ryzhii, I. Semenikhin, M. Ryzhii, D. Svintsov, V. Vyurkov, A. Satou, and T. Otsuji, J. Appl. Phys. 113, 244505 (2013).

[28] D. Svintsov, V. Vyurkov, S. Yurchenko,T. Otsuji, and V. Ryzhii, J. Appl. Phys. 111 (2012) 083715.

[29] F. T. Vasko and A. V. Kuznetsov, Electronic States and Optical Transitions in Semiconductor Heterostructures (Springer, New York, 1999).

[30] H. Shi, H. Pan, Y.-W. Zhang, and B. Yakobson, Phys. Rev. B 87, 155304 (2013).

[31] M. A. Belkin, J. A. Fan, S. Hormoz, F. Capasso, S. P. Khanna, M. Lachab, A. G. Davies, and E. Linfield, Optics Express 16, 3242 (2008).

[32] H. Kanaya, H. Shibayama, R. Sogabe, S. Suzuki, and M. Asada, Appl. Phys. Express 5, 124101 (2012). 